\title{
Imagine No Religion. How Modern Abstractions Hide Ancient Realities
}

New York, 2016, Fordham University Press, pp. 309, ISBN 978-0-8232-7120-7, \$ 125.00 (hardcover), \$ 35.00 (paperback), $\$ 14.39$ (e-book)

\section{Corinne Bonnet}

\section{(2) OpenEdition}

12 Journals

Édition électronique

URL : http://journals.openedition.org/mythos/352

DOI : $10.4000 /$ mythos.352

ISSN : 2037-7746

Éditeur

Salvatore Sciascia Editore

\section{Édition imprimée}

Date de publication : 1 décembre 2018

Pagination : 139-141

ISBN : 978-88-8241-501-3

ISSN : $1972-2516$

\section{Référence électronique}

Corinne Bonnet, «Imagine No Religion. How Modern Abstractions Hide Ancient Realities », Mythos [En ligne], 12 | 2018, mis en ligne le 24 septembre 2019, consulté le 25 septembre 2020. URL : http:// journals.openedition.org/mythos/352; DOI : https://doi.org/10.4000/mythos.352 


\title{
Carlin A. Barton, Daniel Boyarin
}

\author{
Imagine No Religion. How Modern Abstractions Hide Ancient Realities \\ New York, 2016, Fordham University Press, pp. 309, ISBN 978-0-8232-7120-7, \\ \$ 125.00 (hardcover), \$35.00 (paperback), \$ 14.39 (e-book)
}

\section{Corinne Bonnet - Université de Toulouse (UT2J) - cbonnet@univ-tlse2.fr}

Le livre attire d'emblée par son titre; cet Imagine No Religion, qui nous a tous fait rêver et chanter, dans le sillage du grand John Lennon, en 1971. Le sous-titre explicite parfaitement la perspective adoptée : que verrait-on si, pour décrire les pratiques et les conceptions des Grecs et des Romains en rapport avec les dieux, on renonçait au concept moderne de "religion(s) " ? Le défi est considérable et stimulant à la fois. II implique - et c'est précisément l'objet de ce livre important - de revenir sur les notions anciennes, religio en latin, thrēskeia en grec, pour en saisir la complexité diachronique et synchronique. Le volume, écrit à quatre mains, mais parfaitement "amalgamé ", comme le revendiquent justement les auteurs dès la note initiale ( $\mathrm{p} . \mathrm{IX})$, est donc structuré autour de ces deux grandes enquêtes; l'une et l'autre adoptent le même plan: tout d'abord une section intitulée "Mapping the word " où il s'agit de cartographier les usages du terme dans une multitude de contextes, en prêtant attention à ses évolutions ou mutations, à ses multiples facettes, aux champs sémantiques impliqués, aux questions de traduction, etc. ; ensuite une section consacrée à un case study, Tertullien pour religio, Flavius Josèphe pour thrēskeia. On voit comment ces deux approches combinent astucieusement un repérage horizontal, si l'on peut dire, et une analyse verticale, dans toute l'épaisseur d'un témoin, dont l'œuvre abondante et versatile jette une lumière significative sur les "mots et les choses". À bien des égards, en effet, la démarche des auteurs s'apparente à une archéologie du savoir, dans la lignée de Michel Foucault (pourtant absent de la bibliographie, quoique $\mathrm{C}$. Barton se compare à une "archaeologist " au début de son investigation - p. 15). L'Introduction, intitulée What You Can See When You Stop Looking for What Isn't there (p. 1-9), précise néanmoins de manière très solide et efficace les fondements d'une démarche de déconstruction, salutaire sans être iconoclaste. II ne s'agit pas simplement de constater l'inadéquation de " religion " pour rendre religio et thrēskeia, mais d'enquêter sur le réseau de significations qu'une étude approfondie de leurs usages permet de voir. "Religion " est dénoncé ici comme une facilité, un raccourci, mais surtout un écran qui masque la complexité des témoignages anciens sur ce que l'on pensait, ce que l'on disait des dieux, comment on communiquait, comment on interagissait avec eux. II ne s'agit donc pas de démolir l'édifice et de transmettre un paysage en ruines, mais de montrer la variété des phénomènes sociaux que l'on range sous ce "label " moderne et que l'on saisit bien mieux si l'on recourt à la terminologie emic. En particulier, l'idée de force du volume est de montrer que, contrairement à ce que le terme "religion" suggère, héritage de la pensée chrétienne qui s'en est emparé et en a modifié le sens, nous n'avons pas affaire à des faits sociaux séparés, isolés, autonomes, spécifiques, mais à l'inverse à un tissu de pratiques et de représentations profondément imbriquées dans les comportements politiques, économiques, sociaux et culturels.

Retour aux sources : tel est le mot d'ordre. Pour ce qui concerne religio, C. Barton trace fermement une ligne d'évolution qu'elle argumente ensuite, de manière remarquable et passionnante, en cartographiant les usages (p. 15-52): "I will argue that it is exactly the flexible, undefined, and less formalized powers and play of emotions exercised in Latin religio that will be suppressed in an increasingly defined, disciplined, regimented system of government legitimated by reference to a notion of an ultimate authorizing power " (p. 16). II est 
impossible de rendre brièvement compte de toute la palette de nuances, sens, usages que son enquête révèle. Elle montre, de manière très convaincante, le rôle de Cicéron comme pivot dans une certaine orientation du sens de religio en rapport avec un contexte politique turbulent, celui des Guerres civiles, qui demandait une reprise en main des comportements éthiques et politiques, sous l'autorité d'un pouvoir supérieur, porteur d'ordre et source d'un conditionnement psychologique alors jugé indispensable pour le salut de l'État. Avant Cicéron, la notion de religio véhicule, en effet, un ensemble plus complexe de notions, sentiments, comportements et relations avec les dieux. C. Barton examine le lien entre religio et scrupule, hésitation, obligation, tabou, interdiction, retenue, pudeur, anxiété, culpabilité, énergie, etc. Elle montre bien les limites de l'opposition, souvent mise en avant, mais " paralyzing " selon ses termes, entre religio et superstitio. Elle s'arrête ensuite sur le Ciceronian Turn, caractérisé par la nécessité de nouvelles formes de régulation des comportements sociaux, non plus intériorisée, comme c'était le cas jusque-là, mais extérieure, par le biais des institutions, davantage que par un code moral plus ou moins partagé. On trouve ainsi dans le De Legibus cicéronien, des formulations très éclairantes ; par exemple en II, 7, 15-16 (cité p. 49), lorsqu'il s'agit de convaincre les citoyens que les dieux sont les domini omnium rerum ac moderatores, qui scrutent tout un chacun et qui, en tant que membres de la communauté, sont les témoins et les juges de tout ce qui s'y passe. Désormais, ce n'est plus la famille, la maisonnée, les biens qui sont la finalité du comportement religiosus, mais l'État. Cette évolution sémantique, qui associée la religio à une autorité suprême, est amplifiée par les auteurs chrétiens, notamment par Tertullien, étudié de manière très scrupuleuse dans la seconde partie (p. 55-118). Le gigantesque recensement entrepris montre effectivement que la sphère couverte par la notion de religio tend à se distinguer - en restant une nébuleuse - tout en se plaçant sous l'autorité du gouvernement impérial, intermédiaire entre les hommes et le dieu désormais unique et transcendant. C. Barton met en évidence la montée en puissance de connotations déjà présentes, mais qui trouvent dans la pensée chrétienne un point d'aboutissement, en particulier celle de "peur ", a perfect fear, et celle de "discipline " (issue du vocabulaire des courants philosophiques), puisque le chrétien est, de sa propre volonté, un esclave de Dieu. Religio oriente donc vers une soumission totale, en relation avec l'émergence de la notion de conscientia, au sein d'un système rigidement hiérarchisé, une véritable forteresse mentale et politique. L'analyse de $\mathrm{C}$. Barton montre bien ce que les conceptions de Tertullien doivent à un contexte de christianismes bourgeonnant et concurrents, ainsi qu'à la confrontation avec le judaïsme.

Le concept de thrēskeia - un terme dont l'étymologie demeure obscure - subit, si l'on peut dire, le même traitement de la part de D. Boyarin (p. 123-209); le dossier est tout aussi riche d'enseignements, même si le recouvrement entre thrēskeia et religio, d'une part, thrēskeia et religion, de l'autre n'est que partiel, comme le concède Boyarin (p. 123). II souligne aussi le fait que thrēskeia n'est que très rare à l'époque classique, où ce terme s'applique surtout, chez Hérodote notamment, aux pratiques et croyances des "autres, et émerge avec plus de clarté à la fin de l'époque hellénistique, puis à l'époque romaine. Sa valeur heuristique est donc indéniable, même si les spécialistes de l'époque classique pourront éprouver une certaine frustration face à ce choix. Les intersections avec d'autres notions, comme eusebeia, asebeia, deisidaimonia, nomos, latreia, etc. sont dûment prises en compte. Flavius Josèphe, auteur complexe, dont le positionnement est chargé d'ambiguïté, sert de fil conducteur à une enquête passionnante sur la thrēskeia des Juifs, au regard du discours développé parallèlement dans le Nouveau Testament, chez Philon d'Alexandrie et dans 4 Maccabées. II faut partir du constat qu'aucun auteur n'utilise ce terme plus que Flavius Josèphe. II renvoie au service de la divinité, à l'ordo sacrificiel, mais aussi au nomos, pris au sens le plus large, impliquant à la fois un état d'esprit et un comportement en lien 
avec la justice et la fidélité aux engagements. Comme dans le cas de Tertullien, mais pour d'autres raisons, l'ambivalence des usages textuels remonte savamment des analyses et fait obstacle à toute traduction simpliste. C'est que les usages sont aussi déterminés par les publics visés, qui sont toujours multiples, à fortiori chez un auteur à l'esprit divisé comme Flavius Josèphe qui a recours à des stratégies narratives et lexicographiques relevant d'un doublespeak. D. Boyarin prolonge son étude jusqu'à la littérature apologétique du $\mathrm{I}^{\mathrm{er}}$ au

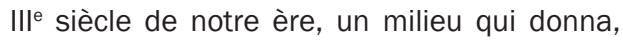
notamment avec Justin le Martyr, la première impulsion à l'émergence d'un vocabulaire marquant la séparation entre le "religieux " et le "politique ".

La conclusion du livre (p. 211-214) revient sur le parti philologique adopté pour l'enquête: quels mots dans quelles strates textuelles? dans quels contextes ? pour exprimer quels contenus?

Le moment est-il venu d'admettre que la catégorie analytique de "religion " appliquée aux cultures de l'Antiquité est fourvoyante, qu'elle brouille plus qu'elle ne clarifie? C'est le pari des auteurs. Le retour aux sources a révélé un réseau relationnel de termes et de notions d'une grande complexité et flexibilité. Les champs qu'ils couvrent impliquent à la fois le registre des représentations, des discours, des pratiques et des émotions. La comparaison entre Tertullien et Flavius Josèphe confirme en tout cas la prégnance du registre du politique sur l'orientation des mots et des choses. En l'occurrence, ce qui se joue, avec religio comme avec thrēskeia, c'est le rapport à l'État, à l'Empire et à l'Empereur, à l'autorité et à la loi, un rapport de collaboration, soumission ou accommodement réciproque.

Ce volume mérite vraiment d'être lu. II est très bien écrit, clair, sans cesse relié aux textes, finement analysés ; il est sensible à la chronologie et aux contextes, intelligemment comparatiste : un vrai travail d'historien. Ce livre est aussi érudit, sans lourdeur, brillant d'un bout à l'autre. La bibliographie finale aurait pu prêter un peu plus attention aux travaux récents en langue française ; I'index est très utile. On sort de cette lecture enrichi et convaincu d'avoir vraiment découvert de nouveaux horizons de réflexion par delà la notion de "religion ". 